\title{
3-Dimensional Indoor Positioning System based on WI-FI Received Signal Strength using Greedy Algorithm and Parallel Resilient Propagation
}

\author{
Shuaib Alam \\ IGIS, National University \\ of Science and \\ Technology, \\ Islamabad, Pakistan
}

\author{
Salman Atif, Ph.D \\ IGIS, National University \\ of Science and \\ Technology, \\ Islamabad, Pakistan
}

\author{
Saddam Hussain \\ National Development \\ Complex, \\ Islamabad, Pakistan
}

\author{
Ejaz Hussain, Ph.D \\ IGIS, National University \\ of Science and \\ Technology, \\ Islamabad, Pakistan
}

\begin{abstract}
The importance of services, based on current location of objects is growing. This is because Global Navigation Satellite System (GNSS) cannot provide object position inside buildings. In modern era the Location Based Services (LBS) are tremendously dependent on Indoor Positioning System (IPS). Parallel RPROP and greedy algorithm were combined for development of IPS using Received Signal Strength (RSS) in heterogeneous environment, the environment comprised of human activity, walls material, cupboards, and various type of surveying machines etc. The propagation of Wi-Fi signal varies directionally, therefore to cope with direction changes in signals; this proposed model produces three sets of weights, which could be considered best for easting, northing and height respectively. Proposed model was trained with $75 \%$ of collected data and tested on remaining $25 \%$ data. Distance error between known points and predicted coordinates was used for accuracy assessment. Through experiments a maximum accuracy of $0.87 \mathrm{~m}$ was achieved and it was found that median error was less than mean error. Median error between known points and predicted coordinates was about $3.32 \mathrm{~m}$ and their mean error was about $4.62 \mathrm{~m}$, which is satisfactory as far as $3 \mathrm{D}$ position determination is concerned. On the basis of results the use of parallel RPROP and greedy algorithm for $3 \mathrm{D}$ position determination in heterogeneous environment is recommended.
\end{abstract}

\section{General Terms}

Indoor Positioning System, Algorithms

\section{Keywords}

WI-FI, Parallel Resilient Propagation, Position Tracking System

\section{INTRODUCTION}

Significance of positioning increased swiftly with the advancement of services to the end users based on their current locations, these kinds of services are known as Location based services (LBS). Location is one of the fundamental component of LBS, provision of such services would not be possible in areas where exact location of the user could not be determined. Location of an object can be determined either using existing communication infrastructure like Wireless Local Area Network (WLAN) or by deploying special infrastructure for positioning such as GPS, RFID, Zigbee etc. It is a general observation that Global Navigation Satellite System (GNSS) does not work inside buildings, since it requires direct line of sight with at least three satellites for reliable position determination. On the other hand deployment of additional infrastructure requires a lot of investment and effort, whereas available communication infrastructure is not only cheap but could be efficiently exploited for position determination as well ${ }^{[1][2][3]}$.

Wireless communication infrastructure is mainly based on wireless local area networks (WLAN). Because of enormous achievements of Wi-Fi in the field of WLAN, Wi-Fi has become a de facto standard for wireless network ${ }^{[1]}$.

The main idea is to utilize signal metrics for location estimation at the desired point of interest. Various signal metrics can be utilized for position determination inside building, like Angle of Arrival (AOA), Time of Arrival (TOA), Time difference of Arrival (TDOA) and Received Signal Strength (RSS). TDOA is the time taken by the signal from transmitter to receiver, distance can be calculated using formula (Frequency X TDOA), AOA is the angle from which signal is received at the receiver. AOA can also be calculated using TDOA. The power of received signal is known as RSS, and this metrics can be utilized reliably for indoor position determination. Every wireless network interface card reports signal strength. Position determination using RSS is both feasible and economical. Based on signal metrics numerous indoor positioning algorithms are available like Proximity Detection, Trilateration, Triangulation, and Fingerprinting.

Using proximity detection algorithm the presence of objects in a certain range can be detected without any physical contact. Proximity detection use Cell of Origin (COA) techniques, which is used for caller position determination. Trilateration on the other hand is a geometrical technique in which absolute or relative position of an object is determined by finding its distance from three points whose coordinates are known.Triangulationis also a geometrical technique in which absolute or relative position of an object is determined by finding its angles from three points whose coordinates are known. Fingerprinting technique involves creation of lookup table. Lookup table consists of RSS at different locations, whose coordinates are known, this lookup table is used for coordinate's determination of location by comparison and analysis of RSS at unknown locations with RSS stored in lookup table.

The location estimation from signal metrics is primarily based on the concept that the signal strength has inverse relation with the distance from AP, and signals have specific pattern at every location in the desired area of interest. But in real time 
environment the behavior of the signals is affected by different factors, for instance human bodies have great influence on signals as human body absorbs signals, signals are also effected by refraction, reflection and multipath ${ }^{[1]}$. So the nature of signals are dependent on the configuration of access points and existence of obstacles like door, glass, human bodies etc., e.g. every wall decreases accuracy up to $1.8 \mathrm{ft}^{[3][6]}$.

Triangulation and trilateration are badly effected by multipath and non-line of sight situation, so both these techniques are not suitable for use in heterogeneous environment. On the contrary, location fingerprinting is a precise location tracking technique and is effective in multipath environment ${ }^{[13]}$.

Fingerprinting are dependent on the efficiency and reliability of pattern matching algorithm. Many pattern matching algorithms exist, which can be classified as probabilistic and deterministic. Probabilistic algorithms are based on statistical computation e.g. Bayesian classification and filtering, however deterministic algorithm looks for exact matching e.g. Nearest Neighbor classifiers and the Neural Network classifier. Mostly, probabilistic algorithm were used for pattern matching, but probabilistic algorithm are not that scalable and memory requirement for such algorithms are too much ${ }^{[3]}$, whereas, ANN efficiently adapt to the changing environment and it is robust against multipath and noise.

\section{LITERATURE REVIEW}

Many IPS ${ }^{[2] ~[5] ~[14] ~ w e r e ~ d e v e l o p e d ~ i n ~ p a s t, ~ b u t ~ g e n e r a l l y ~}$ researchers were focused on 2D location determination, recently focus has been shifted towards position determination with exact floor level.

Gansemer, Hakobyan, Puschel \& Grosmann, (2009) ${ }^{[11]}$, in their experiment used ISO Line and Euclidean algorithm for floor determination and they claimed that using Euclidean distance algorithm they determined exact floor with $100 \%$ accuracy.

Schulcz, Varga \& Tóth (2010) ${ }^{[10]}$, developed IPS by combining empirical propagation model with fingerprinting, they also utilized accelerometer and magnetometer and achieved an accuracy about $3.09 \mathrm{~m}$.

After around three years, $\mathrm{Li}$ et al. $(2013)^{[8]}$, in their study integrated $\mathrm{WiFi}$ and GSM technologies and using K-nearest neighbor algorithm they were succeeded in correct room prediction with an accuracy of $72 \%$. In the same year Bojja, Kirkko-Jaakkola, Collin \& Takala $(2013)^{[9]}$, in their study utilized cost gyro and odometer sensors, and they combined it with a 3D map. Using particle filter, and collision detection, $95 \%$ of the time they predicted accurate floor.

In 2014 Campos, Lovisolo, \& de Campos ${ }^{[12]}$, utilized unsupervised clustering and back-propagation ANN, they achieved floor identification accuracy from 91 97\%, with a $2 \mathrm{D}$ error around $4.5 \mathrm{~m}$.

Since in past most of the research was limited to 2D location estimation with determination of correct floor level, sometime floor identification is not enough, and one need 3D coordinates e.g. a robot in mall need exact 3D location, to place goods in correct shelf or get goods from correct rack. In proposed research efforts were made to develop an efficient algorithm for determination of real $3 \mathrm{D}$ location in heterogeneous environment.

Because of the robustness of ANN in noisy and multipath environment, several researchers developed IPS using Modular Multi-Layer Perceptron (MMLP), a single layer ANN, cascading ANN and genetic algorithm [1] [2] [14]. ANN require selection of suitable learning rate for convergence, which itself is a challenging task, in order to overcome this deficiency, techniques like cascading ANN or combination of Cascading ANN and genetic algorithms were utilized, which increased the complexity of the solution. On the other hand RPROP is considered efficient with respect to other ANN propagation techniques ${ }^{[4]}$.

In 2014, Pessin and his fellow researchers ${ }^{[5]}$ in their study exploited RPROP 2D IPS. On the basis of their research they came to know that ANN and RPROP are dependent on the initial random weights, therefor to overcome this limitation they executed RPROP for several time and then they used the average of these executions and achieved an accuracy of less than a meter.

Massive outlier could influence on optimal selection of RPROP result, therefore in the proposed algorithm the results of the best executions among many parallel executed RPROPs were used, also to cope with directional influence instead of selecting one RPROP, best three were selected by comparing their performance with respect to north, south, and height.

\section{PROPOSED APPROACH}

In this proposed solution, the process was started with the analysis of the nature of signal propagation at certain point, and it was found that the signal strength received from an AP does not remain fixed and fluctuation in unpredictable manner. In order to represent signal strength, median of received signal strength was selected, as it is not affected by large outliers, in the figure $3-1$, the fluctuation in the signal strength of Aps can be easily observed.

Chart Of Signal Strength at a Location

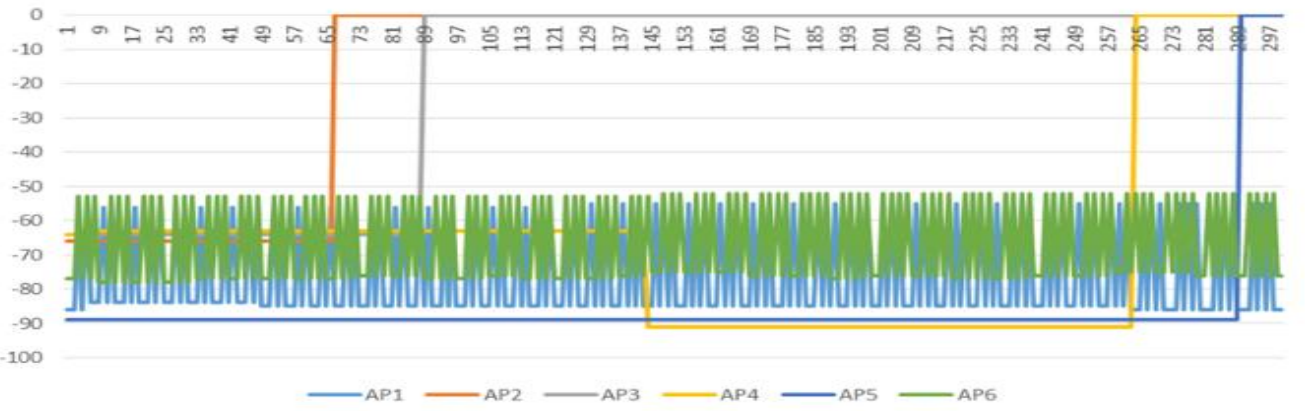

Figure 3-1 Signal strengths received from various access points at a point in the study area. 
Since precision and accuracy of the IPS depends on the number of RSSI samples in the desired study area. For representation of accurate radio propagation, substantial fingerprints data is required ${ }^{[6]}$,also for better performance of ANN and other methods a lot of data is required ${ }^{[8]}$, which requires huge resources, it is a general observation that collection of RSSI samples at all locations in the given study area is not an easy task. In order to meet data requirements, An ArcGIS model to interpolate RSSI for all Wi-Fi access points at 1-foot resolution was developed, since signals also follow directional patterns, therefore for data interpolation Kriging method was utilized.

ArcGIS only support 2D interpolation; therefore RSSI values in accordance to their spot height were grouped. Those RSSI values, whose spot heights were within 1-foot interval, were put in the same group. Since difference between the spot heights within a group is only 1-foot, therefore for every group one unique height in accordance with their spot heights was assigned. Values were interpolated separately for every group.

The ArcGIS model is given in figure 3-2:

In this proposed algorithm three separates weights are generated distinctly for easting, northing and height to cope with directional influence, several RPROP are executed in parallel and then by comparing the results with respect to easting, northing and height, the weights of RPROPs, which are best among them are further optimized.

Complete illustration of the proposed algorithm is given in the figure 3-3:

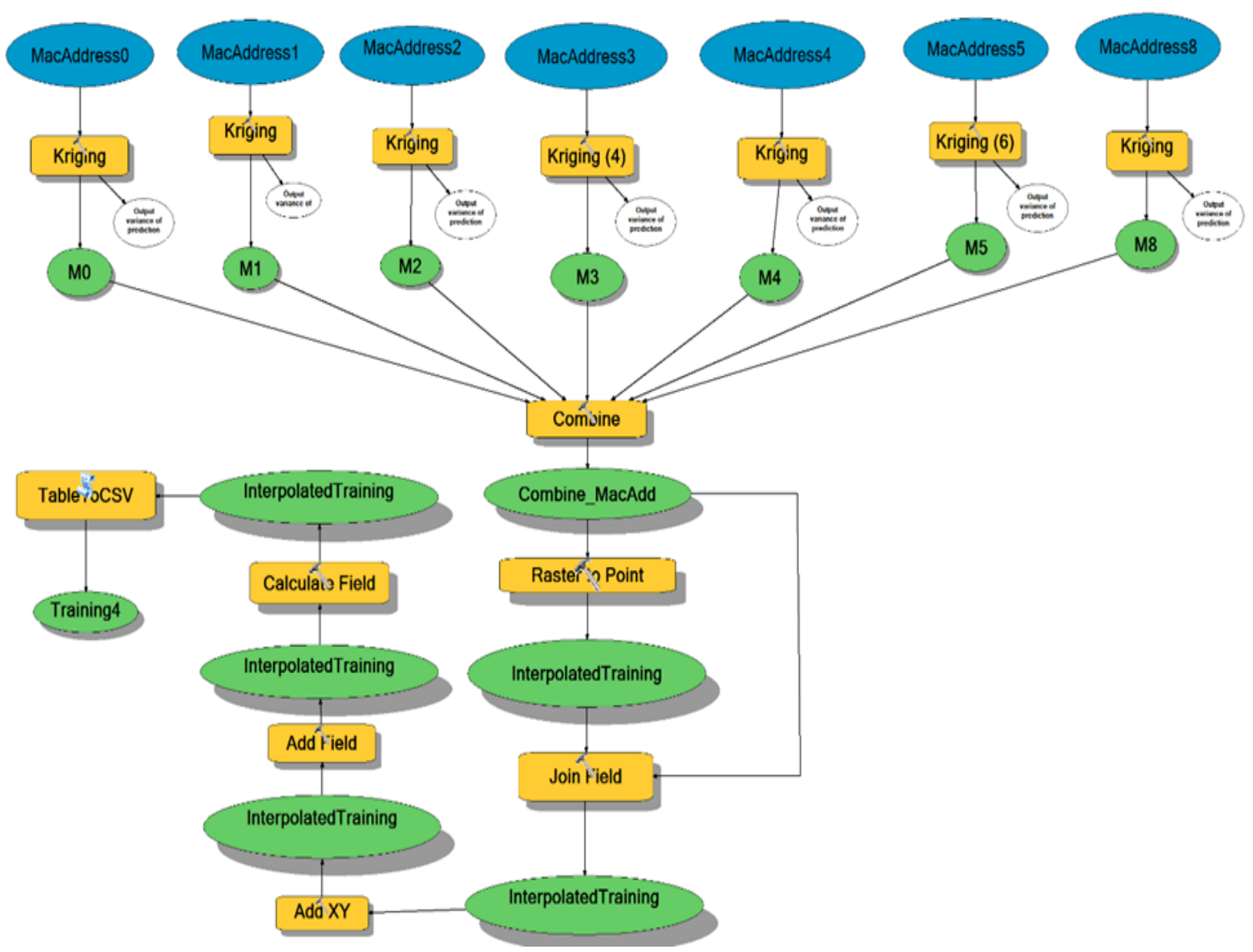

Figure 3-2 ArcGIS model for signal strength interpolation in the study area 


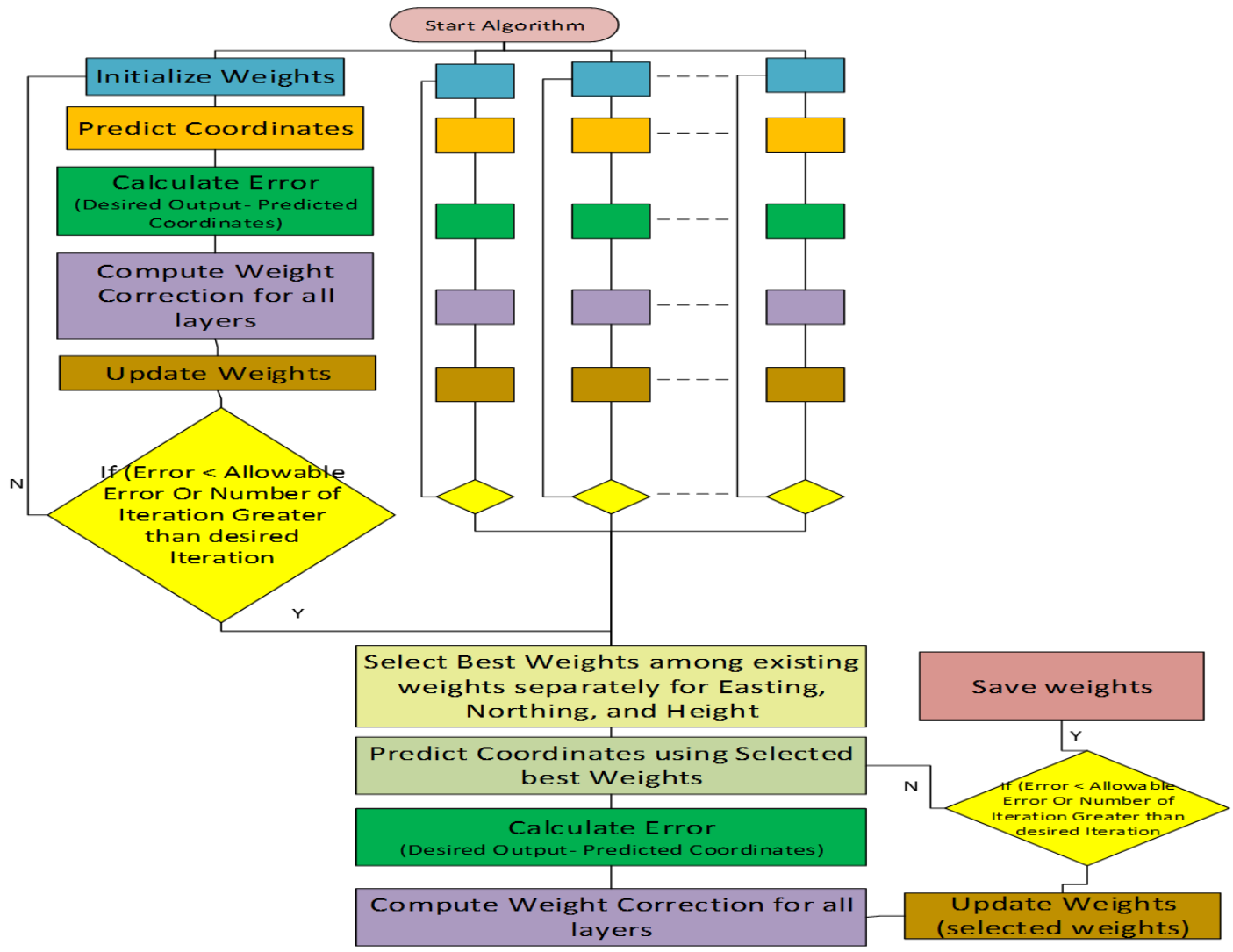

Note: For representation of same process running in parallel, we used same color in algorithm flowchart.

\section{Figure 3-3 Detailed Algorithm Flowchart}

\section{EXPERIMENTAL SETUP AND IMPLEMENTATION OF \\ ALGORITHM}

Proposed system was tested in the survey laboratory at National University of Science and Technology, Islamabad. For Wi-Fi signal strength determination and scanning Alpha adapter (AWUS036H) with 5dbi antenna was used which is of
$802.11 \mathrm{~b} / \mathrm{g}$ standard and Topcon Totalstations GTS-250 Series was used for getting coordinates on known points. Visual studio was used as development platform.

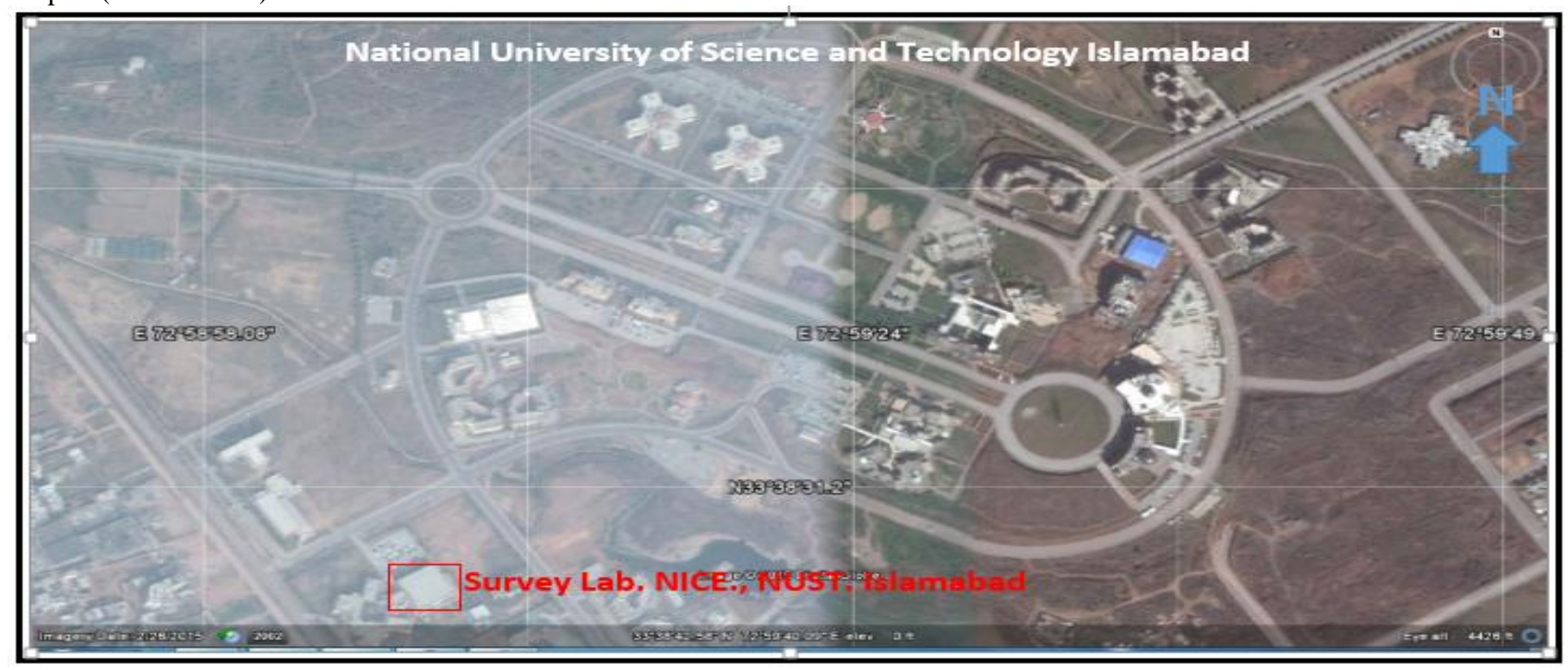

Figure 4-1 Survey Lab. NICE, NUST (Image is taken from Google earth) 


\section{IPDOOR POEITIOAING BYSTIE}

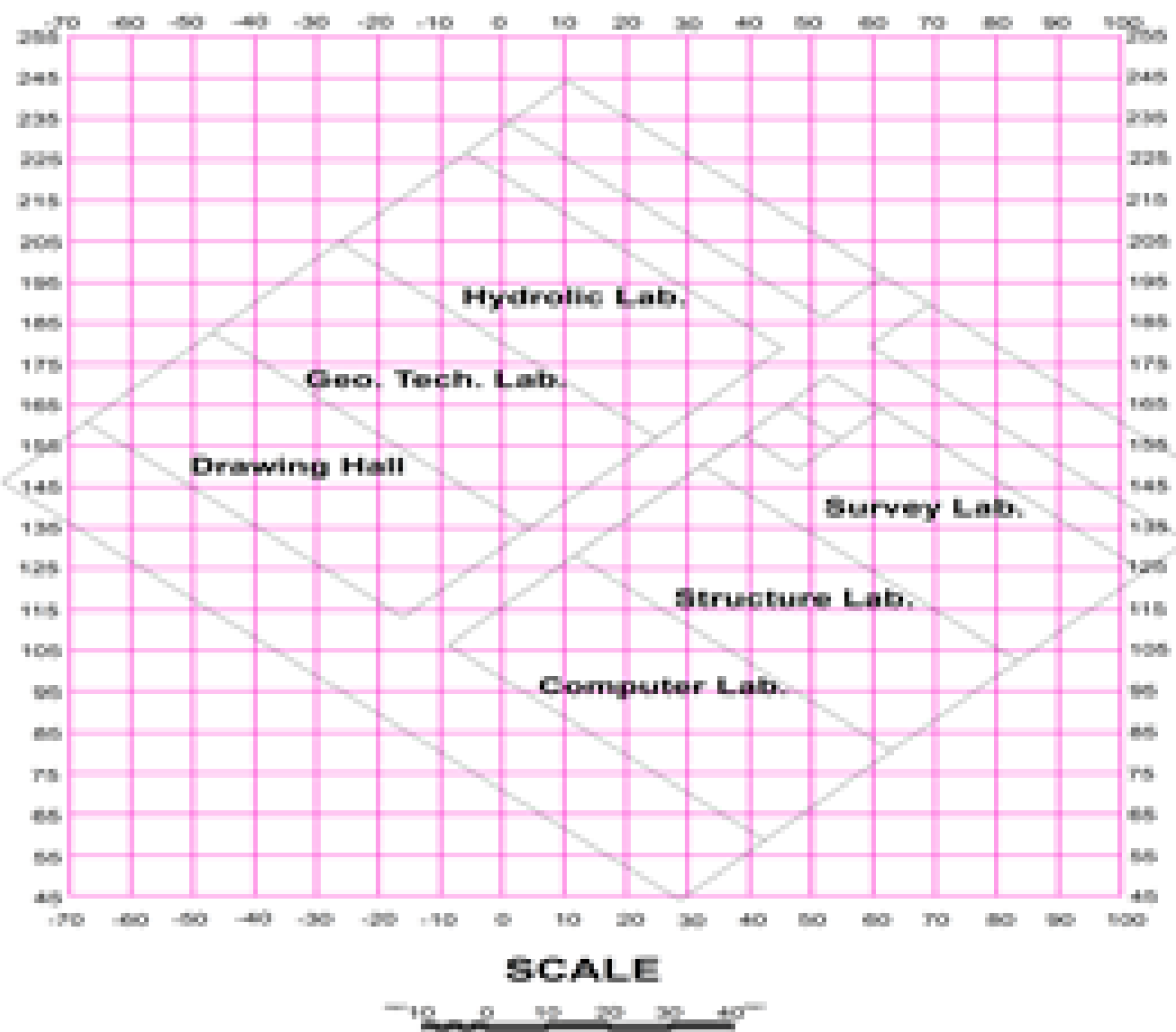

Figure 4-2 Survey Lab. NICE, NUST

\section{RESULTS AND DISCUSSION}

RSSI was observed and collected at about 300 locations, out of which $75 \%$ of the randomly selected data was used for training and the remaining data was used for evaluation of the system. For comparison purposes system was trained using three training algorithms i.e. Clustering using Octree, ANN and RPROP. Through experiments it was concluded that the clustering training algorithm accuracy was about $15 \mathrm{~m}$, which is not sufficient, clustering algorithm had scalability and efficiency issues too, when ANN was used for training, the results were comparatively better than clustering using Octree, but still the error was about $10 \mathrm{~m}$.

Parallel RPROP was quite encouraging as far as 3D environment is concerned, using this method the median error was about 3.71 and the mean error was about $4.6 \mathrm{~m}$. Error graph of Parallel RPROP is given in Figure 5-1,

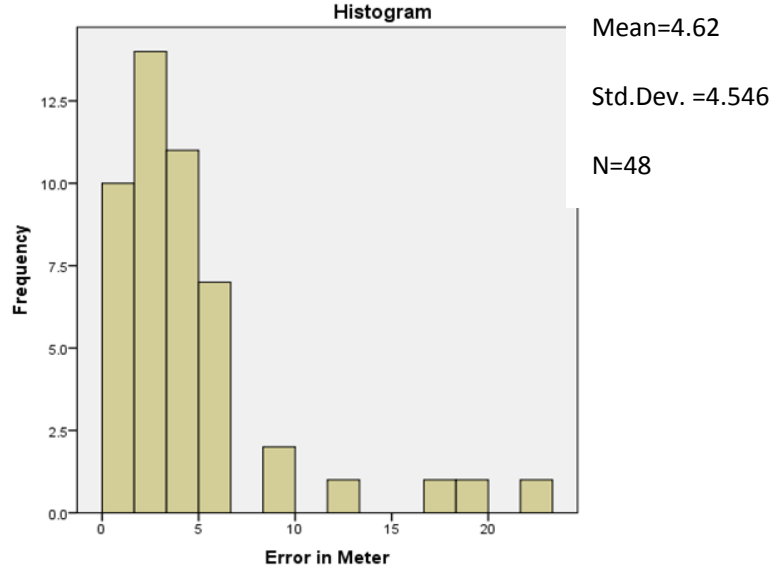

Figure 5-1 Error Graph before generation of data using model

The accuracy of the system was further improved, when data generated from ArcGIS was used for training with parallel RPROP, the median error became $3.13 \mathrm{~m}$ and the mean error became $3.69 \mathrm{~m}$.Error graph of the Parallel RPROP, when 
model generated data was used for training purpose, is given in Figure 5-2.

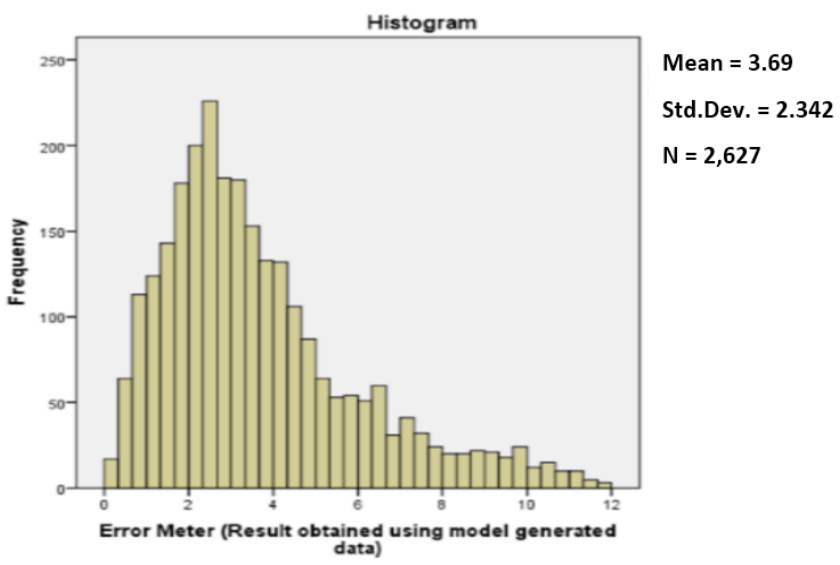

Figure 5-2 Error Graph when model generated data used for training purpose.

\section{Discussion}

By looking at the error map given in figure 5-3, one can observe that error was big near survey laboratory room, where different kind of electronic gadgets were kept. By having a look at the center of the given map, it can be observed that the error is minimal because in this area due to presence of large size table, students' access was limited. Through experiments it was also observed that the presence of the human bodies and electronic gadgets affected the signal strength and hence the accuracy of the proposed system.

By comparing the proposed work with other researcher ${ }^{[8]}[12]$ it can easily be concluded that the results are comparatively more precise and satisfactory (Considering 3-D real time environment). In past researchers were mainly focused on determination of floor level ${ }^{[8][11][12]}$ and $2 \mathrm{D}$ position on that given floor but the focus in the proposed methodology was in exact 3D location determination in real time environment.

\section{CONCLUSIONS}

WI-FI has flourished as the primary standard for wireless LAN. Because of the extensive usage of WI-FI in the industry, government offices and home networks, WI-FI can be easily exploited for positioning systems. It is believed that WI-FI based positioning system would not only be cost effective but it would not require any additional hardware too. WI-FI signal strength were used for the development of IPS based on RPROP, from the results it was concluded that RPROP might be considered as a good choice with respect to cascading ANN, and the resultant system is comparatively more efficient and scalable. By analyzing the results it is recommended that the proposed system should be exploited for practical purposes in different domains.

\section{RECOMMENDATION FOR FURTHER RESEARCH}

The result indicate very promising features of the system, for confidence building the system need to be tested on large scale. Since it is the Era of Android and smart phones so this method need to be exploited for Android platform as well.

Other Artificial Learning Techniques must be used and exploited in this domain. Since Data collection is a very time consuming process therefore a self-mapping mechanism should be developed in order to generate the training data for ANN when it is deployed in new environment.

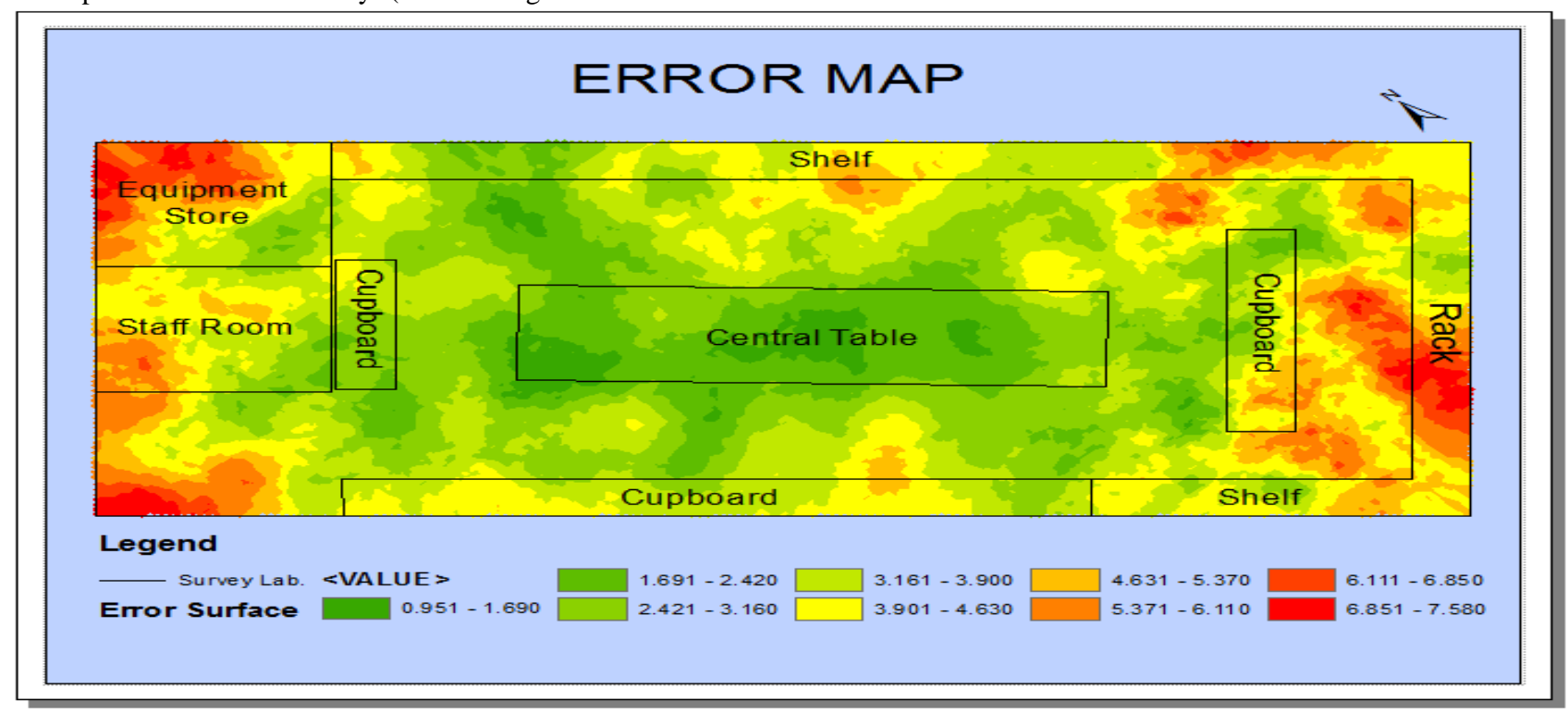

Figure 5-3 Error mapshowing the difference between actual values and predicted values 


\section{REFERENCES}

[1] Ahmad, U., Gavrilov, A., Nasir, U., Iqbal, M., Cho, S. J., \& Lee, S. (2006, April). In-building localization using neural networks. In Engineering of Intelligent Systems, 2006 IEEE International Conference on (pp. 1-6). IEEE.

[2] Mehmood, H., Tripathi, N. K., \& Tipdecho, T. (2010). Indoor positioning system using artificial neural network. Journal of Computer science, 6(10), 1219.

[3] Prasithsangaree, P., Krishnamurthy, P., \& Chrysanthis, P. (2002, September). On indoor position location with wireless LANs. In Personal, Indoor and Mobile Radio Communications, 2002. The 13th IEEE International Symposium on (Vol. 2, pp. 720-724). IEEE.

[4] Riedmiller, M., \& Braun, H. (1993). A direct adaptive method for faster backpropagation learning: The RPROP algorithm. In Neural Networks, 1993., IEEE International Conference on (pp. 586-591). IEEE.

[5] Pessin, G., Osório, F. S., Ueyama, J., Wolf, D. F., Moioli, R. C., \& Vargas, P. A. (2014, March). Selflocalisation in indoor environments combining learning and evolution with wireless networks. In Proceedings of the 29th Annual ACM Symposium on Applied Computing (pp. 661-666). ACM.

[6] Ficco, M., Esposito, C., \& Napolitano, A. (2014). Calibrating Indoor Positioning Systemswith Low Efforts. Mobile Computing, IEEE Transactions on, 13(4), 737751.

[7] Gupta, P., Bharadwaj, S., Ramakrishnan, S., \& Balakrishnan, J. (2014, February). Robust floor determination for indoor positioning. In Communications (NCC), 2014 Twentieth National Conference on (pp. 16). IEEE.

[8] Li, K., Bigham, J., Bodanese, E. L., \& Tokarchuk, L. (2013, April). Location estimation in large indoor multifloor buildings using hybrid networks. In Wireless Communications and Networking Conference (WCNC), 2013 IEEE (pp. 2137-2142). IEEE.

[9] Bojja, J., Kirkko-Jaakkola, M., Collin, J., \& Takala, J. (2013, May). Indoor 3D navigation and positioning of vehicles in multi-storey parking garages. In Acoustics,
Speech and Signal Processing (ICASSP), 2013 IEEE International Conference on (pp. 2548-2552). IEEE.

[10] Schulcz, R., Varga, G., \& Tóth, L. (2010, September). Indoor location services and context-sensitive applications in wireless networks. In Indoor Positioning and Indoor Navigation (IPIN), 2010 International Conference on (pp. 1-10). IEEE.

[11] Gansemer, S., Hakobyan, S., Puschel, S., \& Grosmann, U. (2009, September). 3D WLAN indoor positioning in multi-storey buildings. In Intelligent Data Acquisition and Advanced Computing Systems: Technology and Applications, 2009. IDAACS 2009. IEEE International Workshop on (pp. 669-672). IEEE.

[12] Campos, R. S., Lovisolo, L., \& de Campos, M. L. R. (2014). Wi-Fi multi-floor indoor positioning considering architectural aspects and controlled computational complexity. Expert Systems with Applications, 41(14), 6211-6223

[13] Kolodziej, K. W., \& Hjelm, J. Local Positioning Systems: LBS Applications and Services. 2006.

[14] Borenović, M. N., \& Nešković, A. M. (2009). Positioning in WLAN environment by use of artificial neural networks and space partitioning.annals of telecommunications-annales des télécommunications, 64(9-10), 665-676.

[15] ManagedWI-FI. (2007). Managed WI-FI API. Retrieved March 10, 2013, from http://managedWI-FI.codeplex.com/releases/view/7718.

[16] Heaton,Jeff. (2013). Eencog-dotnet-more-examples-5.3beta2.zip. Encog-cs. Retrieved June 2, 2013 from https://code.google.com/p/encog-cs/downloads/list.

[17] McCaffrey,J. (2012). Dive into Neural Networks. MSDN Magazine. Retrieved April 5, 2013. from http://msdn.microsoft.com/enus/magazine/hh975375.aspx.

[18] Location Map. (n.d.). Retrieved from http://www.nust.edu.pk/AboutUs/ContactUs/Pages/Location-Map.aspx 